\title{
Development of a Mechanistic Model for Wedm
}

\author{
M. Parthiban", A. Prabhukarthi, Naveen Anthuvan \\ PSG College of Technology, Coimbatore, India
}

\author{
*Corresponding Author: M. Parthiban, PSG College of Technology, Coimbatore, India
}

\begin{abstract}
This paper discusses the vibration behavior of the wire to arrive at a general equation for the wire deflection. The movement of wire between upper and lower guide creates vibration; vibration is also created during the machining process. The Finite Element Analysis model [FEA] for the wire deflection is analyzed. Since the general equation is unable to be simplified because of its complex nature, certain assumptions are made to simplify the equation and the obtained partial differential equation is solved by the method of separation of variables to generate a displacement function $y(z, t)$. The displacement function will be able to analyze machining of micro parts, which will be used for further research in micro machining which also helps us to choose the parameters such as current, spark gap, wire feed, and the speed of the work piece, has made its contribution for machining of micro parts with the Wire Electric Discharge Grinding [WEDG]. The FEA results and arrived equation result is compared by equating with some sample values for the equation with various chosen wire frequency.
\end{abstract}

Keywords: FEA, WEDG, Deflection, Vibration

\section{INTRODUCTION}

The Wire Electric Discharge Machining is a non-conventional machining process, in which a conductive wire (tool electrode) travels between the top and bottom guides, in between the work material (also a conductive material- work electrode) is placed. While the powers supplied to the wire, the gap between the wire and the workpiece produces thermal energy and diffuse the work material. The desired shape can be obtained by moving the work table in $\mathrm{X}$ and $\mathrm{Y}$ directions. In the WEDM process, the tool never exerts the force on the work piece so that very hard to machine materials can be machined with ease. The main parameters that influence the production in the WEDM process are spark gap, voltage, current and the wire feed. The decrease in spark gap will lead to increase in the thermal energy, which will result in more material removal. Similarly, the higher voltage will lead to more material removal in the workpiece, which will improve the productivity. The rapid removal of work material may lead to dimensional inaccuracies. In order to maintain dimensional accuracies of the components the voltage, the spark gap and the wire feed has to be proportional. Any complex shape can be easily generated with a high grade of accuracy and surface finish using computer numerical control. Customized software development has further strengthened process technology in improving geometrical and technological data of work piece. Applications of WEDM include manufacturing die and mold components like sheet metal press dies, extrusion dies, etc., prototype and special form inserts manufacturing [5]. Its broad capabilities have allowed it to encompass the production of aerospace/aircraft and medical industries and virtually all areas of conductive material machining An analysis of the effect of various process parameters for achieving improved machining characteristics is required for successful utilization of process with high productivity [2].However, In wire electrical discharge machining (WEDM), the cost of machining is rather high due to- a high initial investment for the machine and the cost of the wire which is used as a tool in this process. The WEDM process is economical if it is used to cut complex work pieces and difficult machine materials While Wire electrical discharge machining (WEDM) has occupied an important position in some high-precision and high-performance manufacturing industries. Due to its capability of accurate and efficient machining parts with varying hardness or complex shapes, the high-machining precision and efficiency, especially at rough corner cutting, cannot be satisfied simultaneously because of some phenomena such as wire rupture, deflection, vibration, etc. [3]. In WEDM process, as the wire moves from the top guide to bottom guide, there will be vibrations 
according to the amount of material removal. Because of vibrations, the spark gap will be varied due to which the material removal rate and the accuracy of the component may get affected. The prediction of the amplitude of vibration of the wire can control the material removal process and the accuracy of the component in WEDM.As newer and more exotic materials are developed, and more complex shapes are presented, conventional machining operations will continue to reach their limitations and the increased use of WEDM in manufacturing will continue to grow at an accelerated rate. [4] Hybrid machining using WEDM started when Masuzawa et. al., and Tongshoff et.al., manufactured small pins, using a rotary axis setup which may be used as a tool three dimensional electrical discharge machining (EDM) applications [5]. Grinding with WEDM is one of the emerging areas developed to generate a cylindrical form on hard and difficult to machine materials by adding a rotary axis to WEDM process [6]. Since our process set up which has been designed and fabricated and kept as an accessory with the existing CNC WEDM for machining of micro parts, it becomes necessary to analyze the vibration response of the wire during the WEDG process. In the WEDG process also the wire never touches the work piece for material removal but as it going to be micro machining process the vibration response may act as a factor in selecting the machining parameters. In order to compute the vibration of the wire, which will exert based on the input voltage, a model was drawn and the same was analyzed with the help of Finite Element Analysis [FEA] software. The force that which the, wire generates based upon the input voltage as given by the research study [7]. Based on this study, the various forces were used for our study in the FEA model and the results were obtained. For the same a mathematical equation has been arrived taking consideration of several factors and both the FEA results and the equation results compared. Since there was no much deviation observed, similar prototype micro-wire EDM setup kept as a reference for the development of our study [8].

\subsection{The Wire-Electrode (Tool) Vibration and The Mathematical Model}

The vibration behavior of the wire is extremely complicated in nature. The occurrence of spark is of highly stochastic in nature, the magnitude and directions of the various forces acting along or upon the wire are not always constant. Moreover, while the wire is in vibration mode, sparks continues to occur in the extreme nodal positions of the wire [9]. The main forces acting along the wire or upon the wire are, forces from the gas bubbles formed by the plasma of the erosion mechanism, hydraulic forces due to flushing, electrostatic force and electro-dynamic force. The hydraulic force will not be significant if the work is submerged in the di-electric medium, hence can be neglected. The wire experiences an impact during every individual spark discharge and acts in the reverse direction of the discharge occurrence. The schematic representation of wire (tool) vibration in WEDM process at normal condition has shown in Figure 1. The forces from the gas bubbles, electrostatic force, electrodynamic force and the axial tensile force of the wire due to tension will induce vibration to the wire when discrete sparks generated between the tool and work piece electrodes. As the wire never touches the work piece there is no induced force during machining and the vibration force may vary depends on the work piece material and the input parameters.

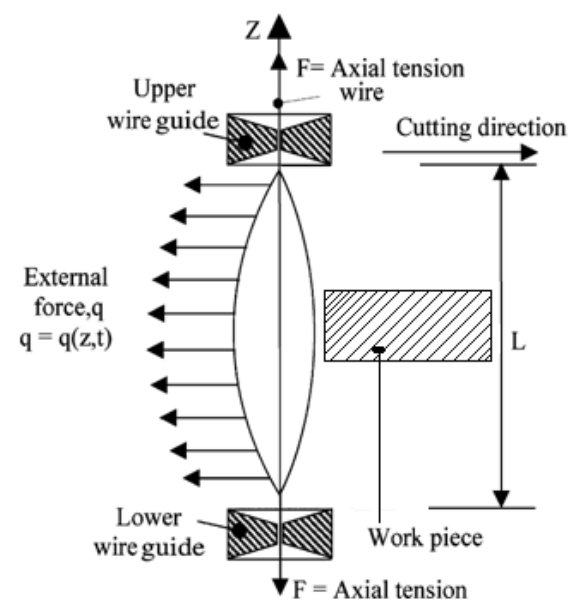

Figure1. Schematic diagram of wire vibration in WEDM [10] 


\subsection{Assumptions ([9], [10])}

The following assumptions was made from the previous studies

1. The wire mass is uniformly spread along its working length.

2. The axial tension remains constant between the wire guides.

3. The wire is static (not moving).

4. The wire is perfectly flexible.

5. The disturbing forces acting per unit length of the wire perpendicular to the axial force varies as a function of time and space.

The general partial differential equation of motion as per Newton's second law of motion for a stretched vibrating string of length $L$ in a plane (along $Z$-axis) is given in equation $1[5,10]$.

$F \frac{\partial^{2} \mathrm{y}}{\partial z^{2}}-E I \frac{\partial^{4} \mathrm{y}}{\partial z^{4}}=\rho \frac{\partial^{2} \mathrm{y}}{\partial \mathrm{t}^{2}}+\beta \frac{\partial y}{\partial t} q(z, t)$

Where, $F$ is wire tension in $(\mathrm{N}) ; y$ is wire deflection in $(\mathrm{m}), t$ is time in $(\mathrm{s}) ; \rho$ is wire mass density in $\left(\mathrm{kg} / \mathrm{m}^{3}\right) ; s$ is wire cross-section in $\left(\mathrm{m}^{2}\right) ; \beta$ is specific damping co-efficient of the wire, $E$ is Young's modulus in $\left(\mathrm{N} / \mathrm{m}^{2}\right) ; I$ is area moment of inertia, $d$ is wire diameter in $(\mathrm{m})$ and $q(z, t)$ is the specific external load or body force per unit length of the wire in $(\mathrm{N} / \mathrm{m})$.

The first term indicates the force due to bending deformation because of the applied axial tension $F$. The second term represents the force required for transverse bending against the flexural rigidity of the wire, as the wire is assumed to be perfectly flexible, this term will be negligibly small. The fourth and fifth terms represent the acceleration force according to Newton's second law of motion and damping force respectively.

The above partial differential equation will give rise to the evaluation of wire deflection, but the partial differential equations are highly complex to solve and the determination of the force $q(z, t)$ is also a difficult task. The analytical solutions for finding $y(z, t)$ is difficult using partial differential equations and requires a wide range of considerations of machining operational features to obtain a real-time solution. However, by using certain simplified assumptions, the complexity of the solution can be reduced and a feasible solution can be arrived by sacrificing a small percentage of accuracy and precision.

\section{Finite ELEMENT ANALYSIS VALIDATION}

In order to make Finite Element Analysis, the CAD model created according to the specification with the use of Creo Parametric 2.0 software. Then the model has converted into Initial Graphics Exchange Specification (IGES) file format, which is the standard format for the importing of the file to finite element software. The material properties of Diamond and Brass were took as input to the Finite element analysis.

The component is discretized into many elements, in order to convert the geometry data into the finite element model. Once the finite model is created, it is then subjected to the boundary conditions. The boundary conditions are given as fixed to the upper guide wire and lower guide wire. Then added to that the displacement is given to the conductive wire i.e. it is made free on $\mathrm{Z}$-axis and the rest two axis are added as ramped data and also the force is applied to the mid-span of the conductive wire.

After applying all the boundary conditions and the load data, the prediction of deformation of the wire electrode for the applying forces as per the samples for $0.002 \mathrm{~N}$ and $0.006 \mathrm{~N}$ force. The deflection of the wire found from $0.0005 \mathrm{~N}$ to $0.006 \mathrm{~N}$ at various levels as given in Table 1 . In order to ensure the attained deformation data for its correctness, the mesh convergence plot been plotted, followed by the applying of forces is done for more trials and the attained deformation value for the respective force is listed in Table 1 and a sample is shown in Figure 2. 




Figure2. Total Deformation for 0.002 N Force

Table1. Total Deformation results from Finite Element Analysis

\begin{tabular}{|c|c|}
\hline Applied Force in N & Total Deformation in mm \\
\hline 0.0005 & 0.1846 \\
\hline 0.0010 & 0.3692 \\
\hline 0.0015 & 0.5538 \\
\hline 0.0020 & 0.7384 \\
\hline 0.0025 & 0.9231 \\
\hline 0.0030 & 1.1077 \\
\hline 0.0035 & 1.2923 \\
\hline 0.0040 & 1.4769 \\
\hline 0.0045 & 1.6615 \\
\hline 0.0050 & 1.8461 \\
\hline 0.0055 & 2.0307 \\
\hline 0.0060 & 2.2153 \\
\hline
\end{tabular}

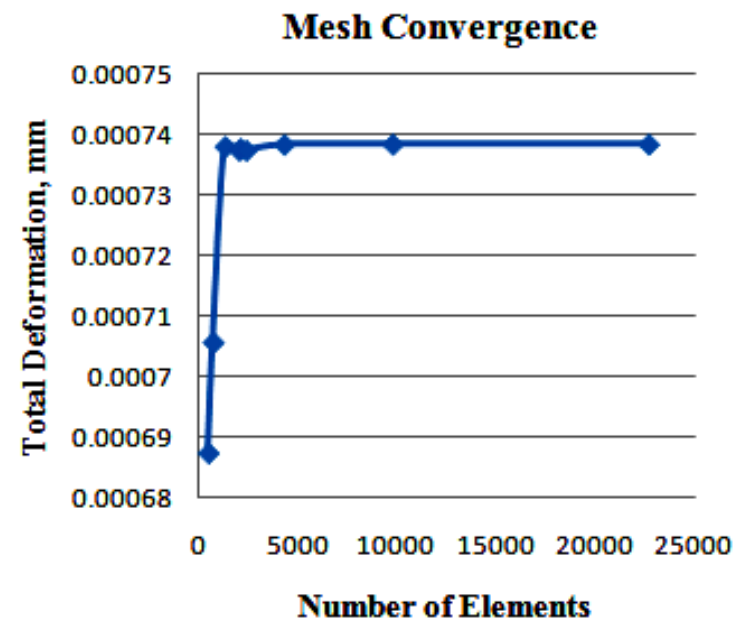

\subsection{Determining Frequency Value}

Modal analysis performed to find out the natural frequency of a model. For the analysis, the model is imported and then assigned to material property (i.e. Brass). After assigning the material property for the geometry model, the model discretized into elements as shown in Figure 5. Then the finite element model is set up with a boundary condition (movement along the $\mathrm{Z}$ - axis made free). After specifying the boundary condition, the variables have been set and the model solved. Then the solution retrieved to find the mode shapes. From this, the frequency range of the model is found out, but in order to predict the resonance conditions (i.e. a frequency at which the component fails) there comes a need of harmonic analysis. In the harmonic analysis, the force (i.e. a point load) is given as an input at the mid-span of the conductive wire. Then the analysis is solved in order to find the frequency response as per Table 2 in $\mathrm{X}$ and $\mathrm{Y}$ axis respectively. From the frequency curve, the maximum amplitude attained for the frequency value of $500 \mathrm{~Hz}$ in $\mathrm{Y}$ direction and $4500 \mathrm{~Hz}$ along X 
direction. Therefore, the obtained frequency value has been set as a reference for the further calculation purpose.

Table2. Frequency Response Values ( $X$ and $Y$ )

\begin{tabular}{|c|l|}
\hline Frequency, $\mathbf{Y}[\mathbf{H z}]$ & Amplitude $[\boldsymbol{\mu m}]$ \\
\hline 500 & 0.1092 \\
\hline 1000 & 0.0131 \\
\hline 1500 & 0.0284 \\
\hline 2000 & 0.0273 \\
\hline 2500 & 0.0089 \\
\hline 3000 & 0.0086 \\
\hline 3500 & 0.0024 \\
\hline 4000 & 0.0007 \\
\hline 4500 & 0.0097 \\
\hline 5000 & 0.0097 \\
\hline
\end{tabular}

\begin{tabular}{|c|c|}
\hline Frequency, $\mathbf{X}[\mathbf{H z}]$ & Amplitude $[\boldsymbol{\mu m}]$ \\
\hline 500 & 0.00025 \\
\hline 1000 & 0.00028 \\
\hline 1500 & 0.00032 \\
\hline 2000 & 0.00024 \\
\hline 2500 & 0.00027 \\
\hline 3000 & 0.00031 \\
\hline 3500 & 0.00029 \\
\hline 4000 & 0.00032 \\
\hline 4500 & 0.00036 \\
\hline 5000 & 0.00031 \\
\hline
\end{tabular}

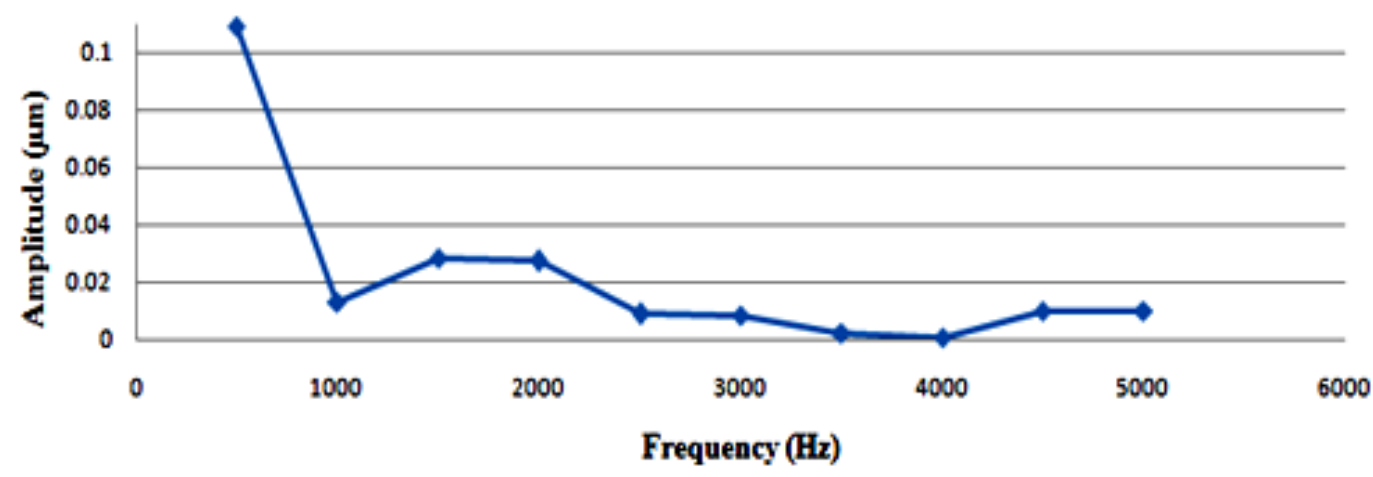

Figure3. Frequency Response Plot - Y Axis

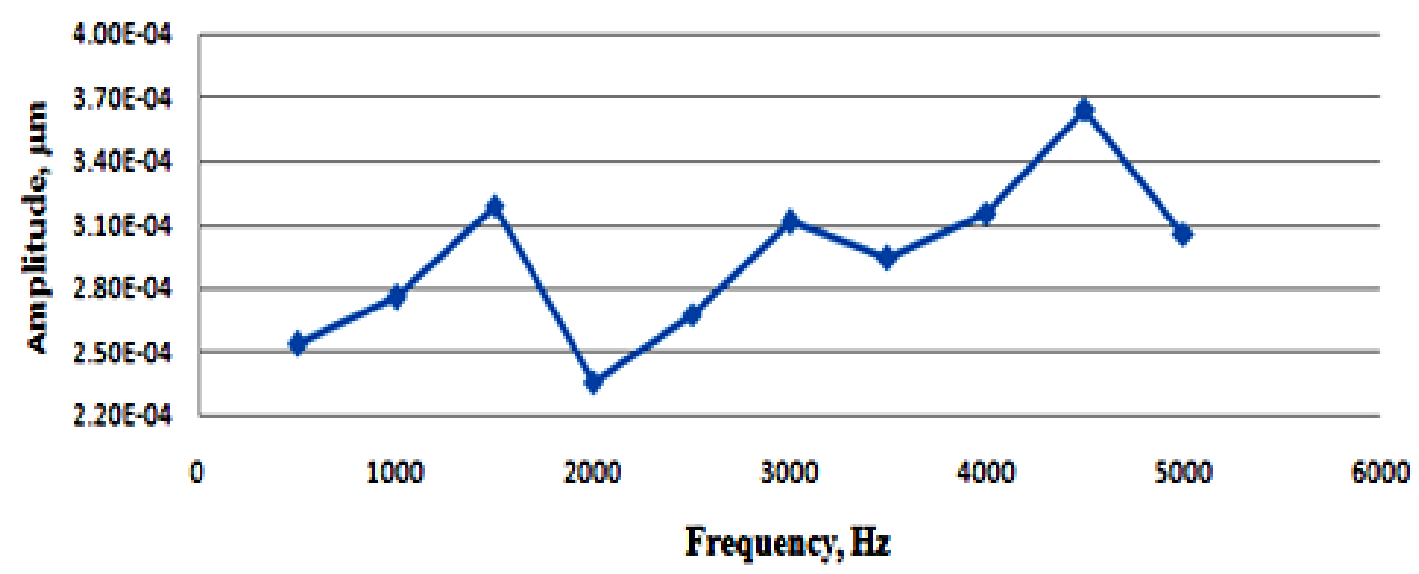

Figure4. Frequency Response Plot - X Axis 


\section{Multi-Discharge Modeling OF WIRE Vibration AND APPROACH TO ObTAin FEASible SOLUTION}

The work piece kept at the mid-position of the wire during machining [11]. The distance between the two wire guides is length $\mathrm{L}$, the length of the wire. The wire is displaced laterally through a distance over the height of work piece $\mathrm{H}$, due to the action of body force $q(z, t)$, for different modes. The body force being the predominant one pushes the wire away from the work piece during induction of the spark discharges. The wire deflects through a distance of $a_{0}$ from its ideal initial stretched condition over the work piece height and the same released at time $t$. The equation of motion, frequency, and the maximum amplitude of vibration of the wire under such condition computed. The frequency responses of the given amplitudes have shown in Figures $3 \& 4$ and values for the same given in Table 2. From the geometry of the wire, as shown in Figure 3, the Initial Conditions (IC) [12] and the assumptions as said above may determine the displacement function $\mathrm{y}(\mathrm{z}, \mathrm{t})$ :

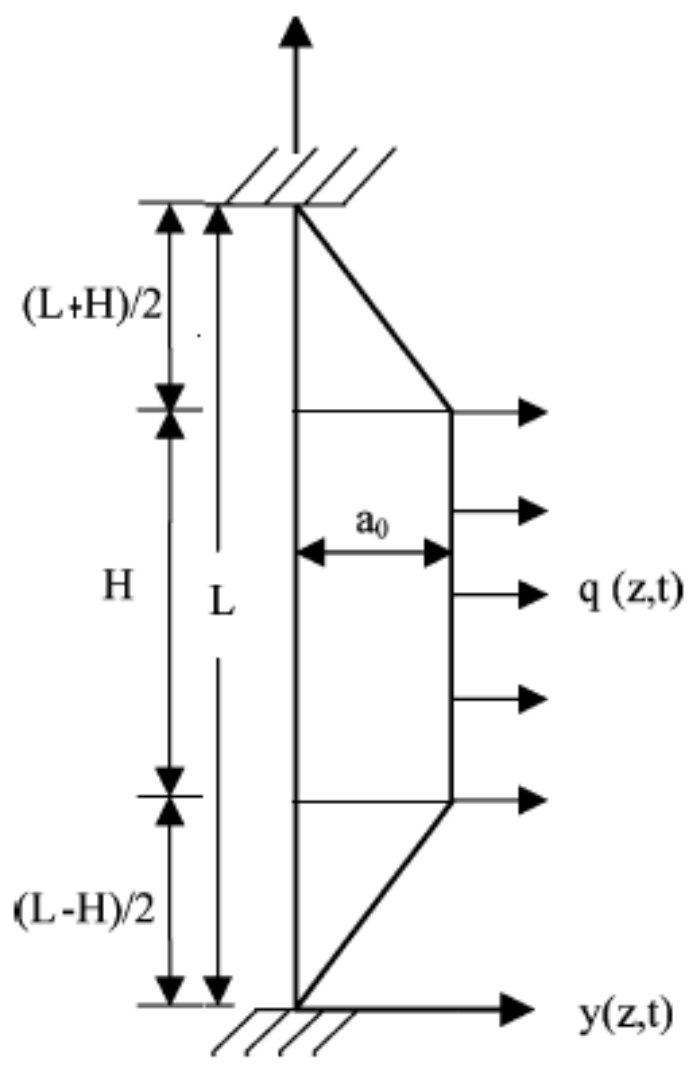

Figure5. Action of the body force before the wire set to vibration. [2, 12]

The Figure 5 shows the maximum deflection induced between the wire guides at the normal condition. The axial tensile force acting on the wire varies gradually, from zero near to the guide and the maximum at the centre. The angle of the deflection ' $\theta$ ' of the wire computed using as per the equation 2.

$$
\tan \theta=\frac{\text { Oppositeside }}{\text { Adjacentside }} \frac{2 a_{o}}{(L-H)}
$$

The angle of deflection of wire in lateral and transverse direction with respect to the lower wire guide computed as per by equation 3 .

$\tan \theta=\frac{y(z t)}{z}$

Equating the (2) and (3), the lateral deflection of the wire computed with respect to time (equation 4)

$Y(z, t)=\frac{2 \mathrm{a}_{0} \mathrm{z}}{(\mathrm{L}-\mathrm{H})}$

Similarly, the lateral and transverse direction with respect to the upper wire guide position computed as per equation 5 .

$Y(z, t)=\frac{2 \mathrm{a}_{\mathrm{o}}(\mathrm{L}-\mathrm{z})}{(\mathrm{L}-\mathrm{H})}$ 
From the geometry of the wire (Figure 5), the displacement function $y(z, t)$ determined by the following initial conditions (ICs):

IC $: \mathrm{y}(\mathrm{z}, 0)=f(z)$

$$
= \begin{cases}\frac{2 \mathrm{a}_{0} \mathrm{z}}{\mathrm{L}-\mathrm{H}} & \text { when } 0 \leq \mathrm{z} \leq \frac{\mathrm{L}-\mathrm{H}}{2} \\ \mathrm{a}_{\mathrm{O}} & \text { when } \frac{\mathrm{L}-\mathrm{H}}{2} \leq \mathrm{z} \leq \frac{\mathrm{L}+\mathrm{H}}{2} \\ \frac{2 \mathrm{a}_{\mathrm{o}}(\mathrm{L}-\mathrm{z})}{\mathrm{L}-\mathrm{H}} & \text { when } \frac{\mathrm{L}+\mathrm{H}}{2} \leq \mathrm{z} \leq \mathrm{L}\end{cases}
$$

The partial differential equation (Equation 1) may also rewritten as

$\frac{\partial^{2} \mathrm{y}}{\partial t^{2}}=c^{2} 0 \frac{\partial^{2} \mathrm{y}}{\partial \mathrm{z}^{2}}-K \frac{\partial y}{\partial t}-\mathrm{Q}$

Where $c^{2}=\frac{F}{\rho}, K=\frac{\beta}{\rho}$, and $Q=\frac{q}{\rho}, q=q(z, t)$. The body force $(Q)$ constitutes all the forces such as, forces from the gas bubbles formed by the plasma of the erosion process, electrostatic force, electro-dynamic force, hydraulic force, and impact on the wire. The hydraulic force assumed to remain constant for a particular machining condition and setup. Thus, for an iso-energic pulse generator, the body force $(Q)$ expressed as in equation 8 .

$$
Q=f\left(E_{p}, v\right)
$$

Where ' $E_{\mathrm{p}}$ ' is, the energy contained in a pulse or pulse energy, and ' $v$ ' the pulse frequency. If the pulse energy maintained consistently during a machining process, the body force $Q$ expressed in the terms of the discharge frequency. Thus, although a universal relation is extremely difficult to establish for body force, $Q$ written as (equation 9)

$\mathrm{Q}=\mathrm{K}_{1} v^{\mathrm{A}}$

Where, $K_{1}$ is a constant of proportionality in the above relation and $A$ is the constant index for the machining system, having constant hydraulic force in the machining gap and offering constant pulse energy during the process. It is also clear that the amplitude of vibration will go on increasing with the increase of $Q$ and decrease with the wire tension. If the wire tension remains un-changed during a machining process, the displacement function $y$ expressed as follows:

$\mathrm{Q}=\alpha \mathrm{y}$

Where $\alpha$ is the constant of proportionality.

Although it may appear apparently that equation 11 is overly simplified, the complexity lies in determining $\alpha$. From the above relations, the displacement function $y$ expressed for a specific machining condition such spark gap, voltage, and pulse on and off time.

$y=\mathrm{K} v, \mathrm{~K} \frac{\mathrm{k}_{1}}{v}$

The constant $K$ may be determined through experimentation and calibration curves and once $K$ known, $\alpha$ may found. Therefore, Eq. (7) reduced to a linear homogeneous equation as expressed below:

$\frac{\partial^{2} \mathrm{y}}{\partial t^{2}}=c^{2} \frac{\partial^{2} \mathrm{y}}{\partial \mathrm{z}^{2}}-K \frac{\partial y}{\partial t}-\alpha \mathrm{y}$

The above linear homogenous equation solved by the method of separation of variables $[12,13]$ with the following initial conditions (ICs) and boundary conditions (BCs)

(1)IC: $y(z, 0)=f(z)$

(2) $\frac{\partial y}{\partial t}(z, 0)=0$

(3) $y(0, t)=0$

(4) $y(L, t)=0$

The displacement function $\mathrm{y}=\mathrm{y}(\mathrm{z}, \mathrm{t})$ can be computed by solving the equation 12 , 


$$
\begin{gathered}
y(z, t)=e^{-\beta t / 2 \rho} \sum_{n=1}^{\infty}\left\{A \cos \left(\omega_{n} t\right)+B \sin \left(\omega_{n} t\right)\right\} \sin \left(\frac{n \pi}{L} z\right) \\
A=\frac{8 a_{0} L}{n^{2} \pi^{2}(L-H)} \sin \left(\frac{n \pi}{2}\right) \cos \left(\frac{n \pi}{2} \frac{H}{L}\right) \\
B=A \frac{\beta}{2 \rho} \frac{1}{\omega_{n}} \omega_{n}=\frac{1}{2} \sqrt{\left(\frac{4 n^{2} \pi^{2} F}{\rho L^{2}}-\frac{\beta^{2}}{\rho^{2}}+4 \alpha\right)}
\end{gathered}
$$

Where, $\omega_{n}$ is the natural angular frequency of the wire vibration. The above equation (13) is derived from the partial differentiation method $[12,13]$ where A and B are the constants for the amplitude of vibration of wire based on its angular frequency.

In order to verify Finite Element Analysis results and the Mathematical equation, a few sample calculations with the angular frequency of wire been done and shown in Table 3.

Table3. Comparison of Total deformation values

\begin{tabular}{|c|c|c|c|}
\hline \multirow{2}{*}{ Force , $\mathbf{N}$} & \multicolumn{2}{|c|}{ Total deformation value arrived, $\boldsymbol{\mu m}$} & \multirow{2}{*}{ \% Deviation } \\
\cline { 2 - 4 } & Finite Element analysis & Mathematical model & 2.55 \\
\hline 0.0020 & 0.7384 & 0.7577 & 2.56 \\
\hline 0.0025 & 0.9231 & 0.9475 & 2.37 \\
\hline 0.0030 & 1.1077 & 1.1346 & 2 \\
\hline
\end{tabular}

\section{CONCLUSION}

- The equation for amplitude motion of wire may be determined as given by Equation (13).

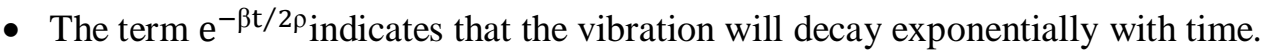

- The maximum amplitude of vibration is $\left(\mathrm{A}^{2}+\mathrm{B}^{2}\right)^{1 / 2}$ and the angular or circular frequency will be $\omega_{\mathrm{n} .} \omega_{1}, \omega_{2}, \omega_{3} \ldots$ are the natural frequencies for $n=1,2,3$. Respectively.

- The fundamental frequency of the vibration will be $\frac{\omega n}{2 \pi}(\mathrm{c} / \mathrm{s})$ for $n=1$.

- The FEA model done and the same has been compared mathematically for some sample values and for $0.0025 \mathrm{~N}$ force and found the deviation is $2.56 \%$ depicts the correctness of the arrived equation.

\section{REFERENCES}

[1] Gokler, M. I., Ozanozgu, A. M.(2000), "Experimental investigation of effects of cutting parameters on surface roughness in the WEDM process", International Journal of Machine Tools \& Manufacture, 40,1831-1848

[2] Parmeswara, C.V.S., and Sarcar, M.M.M. (2009), "Evaluation of optimal parameters for machining brass with wire cut EDM", Journal of Scientific and Industrial Research, 68, 32-35.

[3] Hsue, W.J., Liao, Y.S., Lu, S.S. (1999), "Fundamental geometry analysis of wire electrical discharge machining in corner cutting”, International Journal of Machine Tools \& Manufacture, 39, 651-667.

[4] Spedding, T. A., Wang, Z.Q. (1997), "Study on modeling of wire EDM process", Journal of Materials Processing Technology, 69, 8- 28.

[5] Masuzawa.T, Fujino.M, Kobayashi.K(1985) "Wire electro-discharge grinding for micromachining", CIRP Annals, 34:1: 431-434.

[6] Uhlmann.E, Piltz.S, Oberschmidt.D,(2008), "Machining of micro rotational parts by wire electrical discharge grinding", Prod. Eng. Res. Devel. 2:227-233

[7] Herrero. A et al, (2008) Influence of Force Components on Thin Wire EDM

[8] Yan M.T., Huang C.W., Fang C.C., Chang C.X. (2003), "Development of a prototype micro-wire EDM machine", J.Mater. Process Technol 149:99-105

[9] Jain V K., 2010," Introduction to micromachining", Narosa, India.

[10] CMTI, 1982, “Machine Tool Design Handbook,” Tata McGraw-Hill, India.

[11] Chris Morgan, Shelby Shreve, Ryan Vallance..R, (2003), "Precision Of Micro Shafts Machined With Wire Electro-Discharge Grinding", Proceedings of the ASPE Winter Topical Mtg on Machines and Processes for Micro-Scale, Meso-Scale Fabrication, Metrology and Assembly 
[12] Calin Denes., 2015, “An Overview of Wire Electrode Vibrations on WEDM” Non Conventional Technologies Review, Romania.

[13] Veerarajan.T., 2006, "Engineering Mathematics" Tata McGraw-Hill(Education) India

Citation: M. Parthiban, et.al, (2019)'Development of a Mechanistic Model for Wedm", International Journal of Modern Studies in Mechanical Engineering, 5(3), pp. 1-9. DOI: http://dx.doi. org/10.20431/24549711.0503001

Copyright: () 2019 Authors, This is an open-access article distributed under the terms of the Creative Commons Attribution License, which permits unrestricted use, distribution, and reproduction in any medium, provided the original author and source are credited. 